\title{
Severe Spinal Cord Insult after Reverse Shoulder Arthroplasty
}

\section{Eva García-Jarabo*, Juan María Pardo-García, Leandro Manuel Ramos-Ramos and Manuel Ramón García-Rayo Rodriguez-Barbero}

\author{
Department of Orthopaedics Surgery, Hospital Universitario 12 de Octubre, Madrid, Spain
}

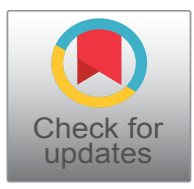

*Corresponding author: Eva García-Jarabo, Department of Orthopaedics Surgery, Hospital Universitario 12 de Octubre, Avenida de Córdoba, 28041, Madrid, Spain, Tel: (+34)-91-3908-39

\begin{abstract}
We present a case of a cervical spine cord ischemia with hemorrhage in a patient with a proximal humeral fracture treated with reverse shoulder arthroplasty (RSA), a rare complication, non-well described in the current literature.

The aim of this study is to review immediate neurological post-operative complications of RSA that can be related to surgery or anesthetic technique. Thus, we describe a severe, rare central system neurological complication after RSA.
\end{abstract}

\section{Keywords}

Reverse shoulder arthroplasty, Complications, Cervical spine cord injury, Beach chair position, Interscalene block anesthesia

\section{Introduction}

Proximal humeral fractures (PHFs) in patients over 65 -years-old are very frequent fractures. Conservative treatment, consisting in sling immobilization and early rehabilitation, stands as "gold standard". Besides, treatment of three and four-part PHF in elderly patients is not well protocolized due to the lack of clear evidence showing better results with surgical treatment [1].

Reverse shoulder arthroplasty (RSA) was initially developed to treat massive rotator-cuff tear osteoarthritis. Further indications to RSA are: PFHs in patients over 75-years-old with functional demands, osteopenia and rotator cuff insufficiency, so as younger patients with complex PHF and rotator-cuff insufficiency where hemiarthroplasty has obtained poor functional results and a high percentage of revision surgery [2].

Due to the increase number of indications, RSA pro- cedures have been increasing exponentially in the last years [3] and associated complications have increased too [2-4].

\section{Case Report}

In 2018, an independent for daily activities 83-year-old female with clinical history of breast cancer, ischemic heart disease and RSA in her right shoulder done 5 years before because of a PHF, was diagnosed at the emergency room with a four-part left PHF (Figure 1).

Surgical treatment was proposed to treat her fracture. In a beach chair position, RSA with tuberosity repair through a transdeltoid approach was performed. Anesthetic technique was: Interscalene block plus general anesthesia consisting in endotracheal intubation (ETI). The parameters monitoring during surgery was blood pressure, oxygen saturation, bispectral index (BIS), electrocardiogram and hydric balance. During anesthetic induction, we highlight an episode of hypotension requiring administration of a single bolus of ephedrine. Neither further action nor other vasoactive drugs were needed. We do not report any outstanding facts surgery-related. Surgery was therefore performed uneventfully.

Patient's physical exam (PE) in the first postoperative day, showed plegia and arreflexia (0/5) in the whole left upper limb. Solely, she was able to initiate finger and fist abduction-adduction. Gait disorder, right neck deviation and ptosis without myosis were as well described during PE. She also complained about global hypoesthesia, especially in the $\mathrm{C} 5$ dermatomal region. 

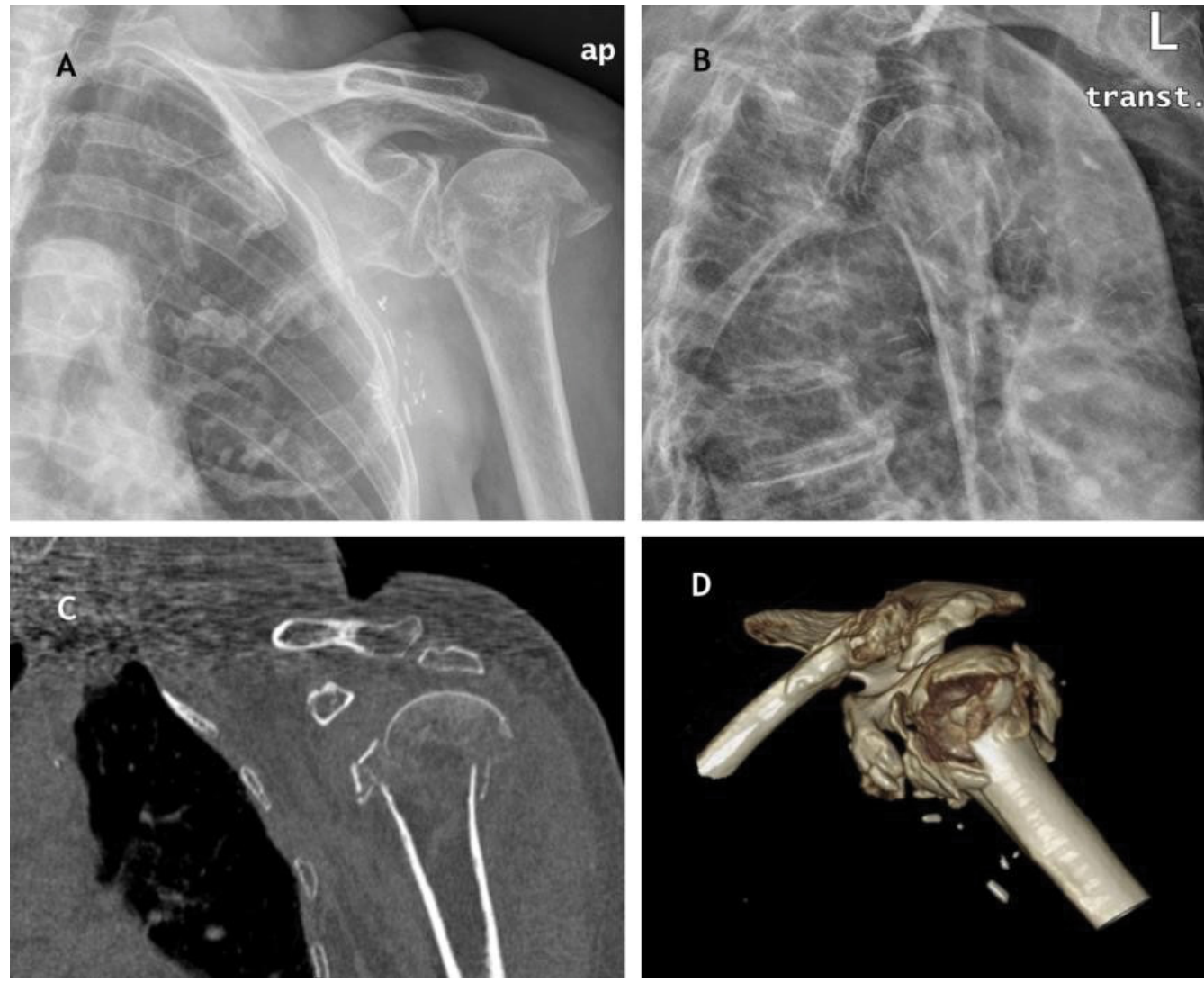

Figure 1: $(A, B)$ Anteroposterior and transthoracic radiographic views. A four-part left proximal humeral fracture; (C, D) Coronal and 3D CT-scan reconstructions.
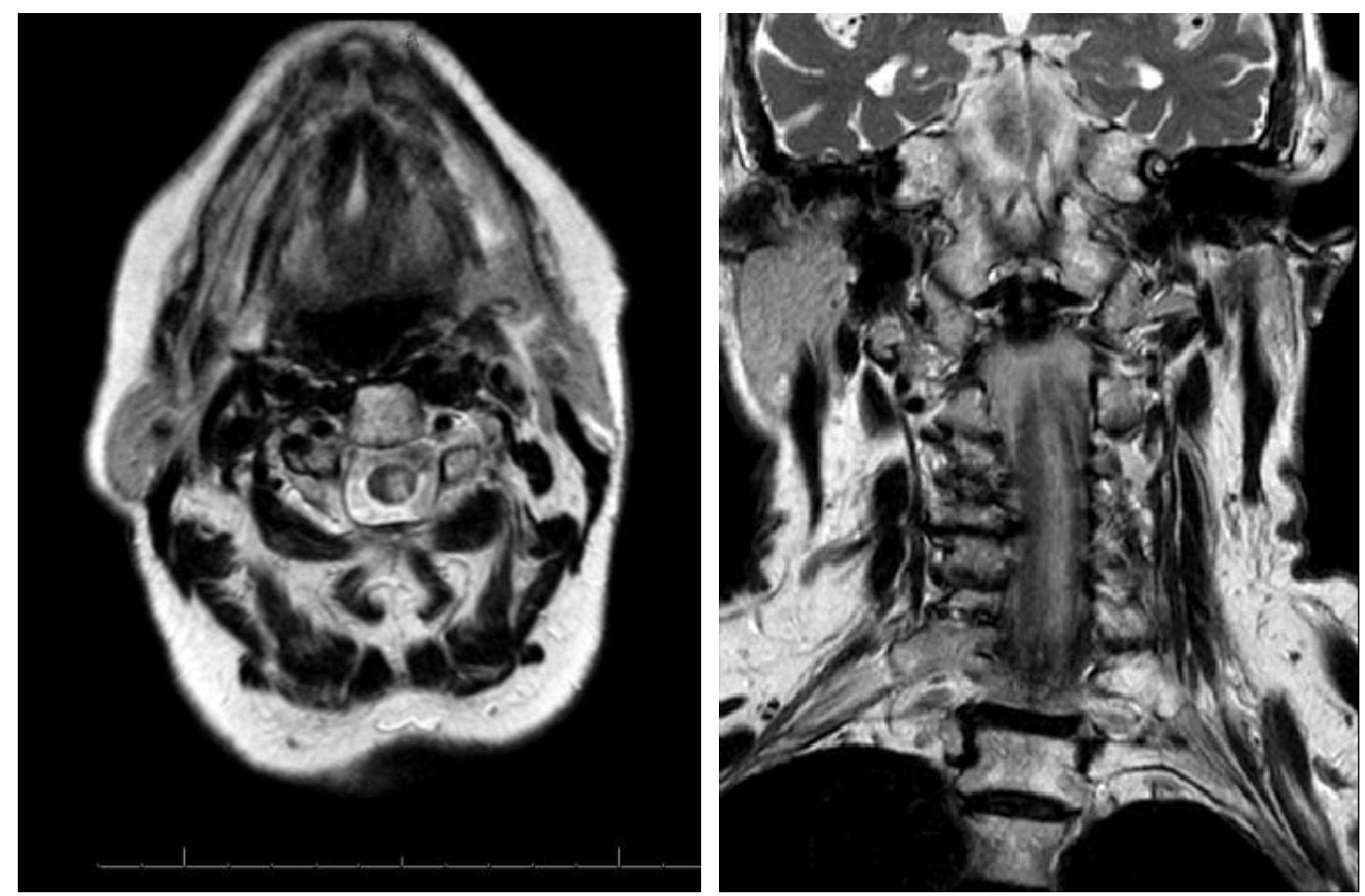

Figure 2: Postoperative MRI, T2 sequences. Hemorrhage component between C1 and C7 limited to left hemimedulla affecting to posterior and lateral tracts.

An urgent magnetic resonance imaging (MRI) of the cervical spine and an urgent evaluation by a neurologist were requested.

MRI images showed: Ischemic tissue damage, with a hemorrhage component between $\mathrm{C} 1$ and $\mathrm{C} 7$ limited to left medulla (Figure 2).

There was no evidence of brachial plexus or vertebral artery injuries. As Neurology team recommenda- 

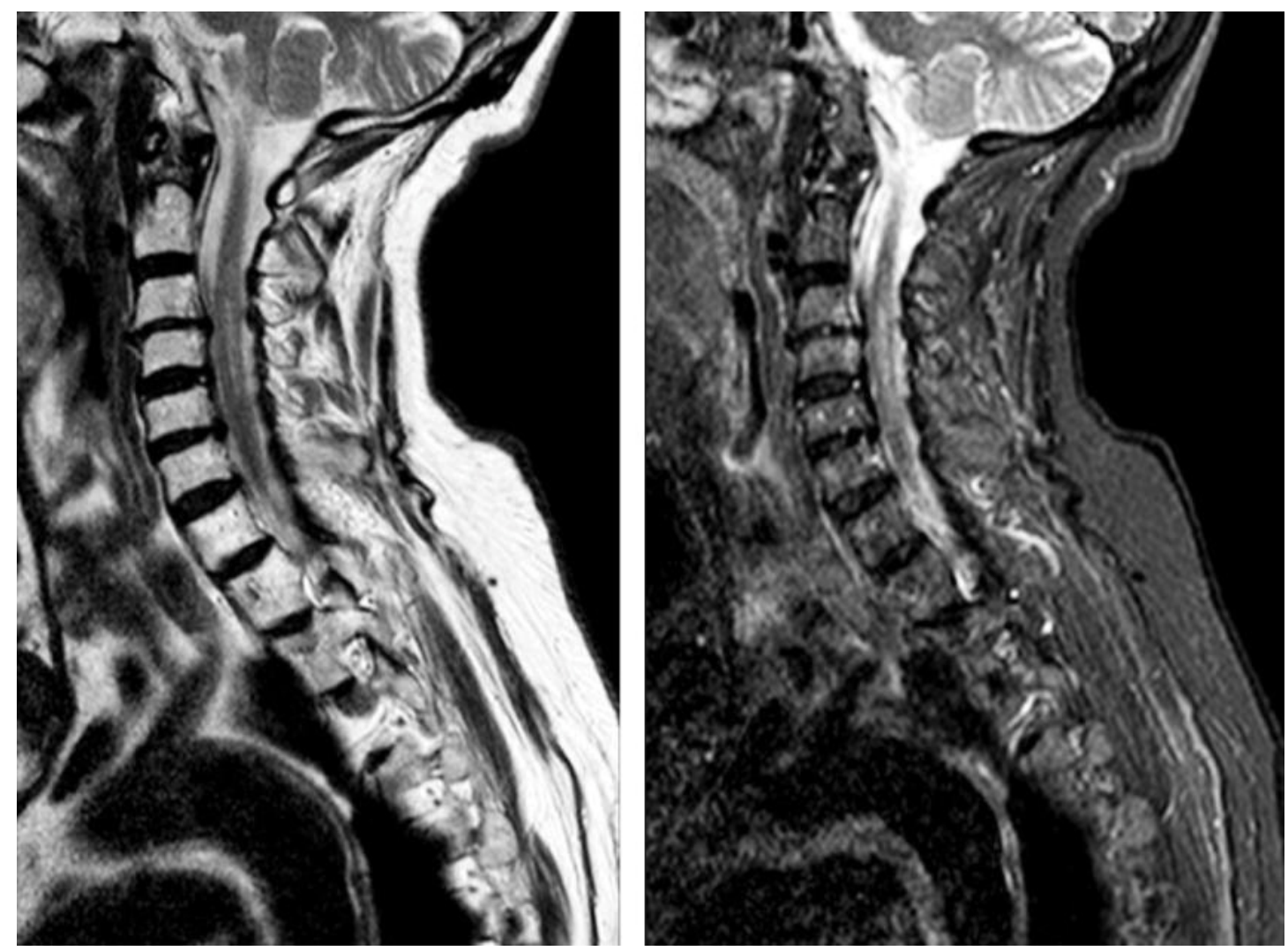

Figure 3: 2 months postoperative MRI. Longitudinal injury between C2 and C7. Hiperintense in T2 (A) and STIR (B) sequences.

tion, a low-corticosteroid dose treatment consisting in four milligrams of Dexamethasone every eight hours for a week and prompt rehabilitation, using a Muenster orthosis, were initiated. Also she took acetylsalicilyc acid $100 \mathrm{mg}$. To complete neurological examination, a complementary electromyogram (EMG) was carried out at one month postoperative. EMG's results reported preganglionic motor neurons lesion, between C3-C4 and T1, especially at C5 and C6 levels. All sensory modalities (pain, temperature, vibration and proprioception) were affected. Moreover, a traumatic radial neuropathy was detected.

A clinical review was taken monthly during the first six months. Progressive, gradual slow but good recovery was observed whereas the patient kept on rehabilitation program. Follow-up MRI at 2 months (Figure 3) showed improvement compared to the previous. Follow-up EMG at one year reported C5 and C6 proximal muscle reinnervation.

Final clinical review at 20 months postoperatively, the patient shows passive elbow, wrist and fingers mobility. Shoulder active range of motion of: $45^{\circ}$ of flexion achieving $90^{\circ}$ passive, active abduction of $45^{\circ}$ and passive abduction $80^{\circ}$ with severe limitation of external and internal rotation.

Motor examination according to muscle strength Oxford score, showed deltoid $2 / 5$, triceps $4 / 5$, wrist extensors $3 / 5$, volar flexors $3 / 5$, fingers flexors $3 / 5$, extensor pollicis longus $3 / 5$. Sensitive examination: Maintai- ned global hypoesthesia in the whole left upper limb. DASH score: 75.8 .

Despite of our patient's severe cervical cord injury, she did not hopefully develop any other systemic complications such as cardiovascular or respiratory. She has shown progressive improvement during clinical follow-up due to rehabilitation. Otherwise, we do not expect any further improvement.

\section{Discussion}

Complications rates of RSA vary between authors and surgery type: primary or revision procedures, ranging from $13 \%$ to $68 \%[2,3]$. The most frequent complications are: instability (16.7\%), infection (6.7\%), intra-operative periprosthetic fracture $(3 \%)$ and neurological injuries, witch its incidence varies from 1 to 4\% [4].

On one hand, among neurological insults, neurapraxia are more frequent than complete nerve injuries (axonotmesis), affecting especially axillary nerve and the brachial plexus. These injuries could happened as a result of surgical approaches, prosthetic components implantation, direct nerve damage during surgical dissection, excessive traction of the upper limb during exposure or reduction, compression secondary to retractors and postoperative hematoma [5-7]. It is known that the glenoid exposure is the most decisive step preventing nerve insults, especially when we have the arm positioned in abduction, external rotation and extension $[6,8]$. In our case, the radial nerve injury could 
occur during the surgery because of an unaware, unreported incident.

Once a nerve injury is detected at the immediate postoperative period, an EMG should be performed at six weeks from the onset of symptoms to determine the type of neurological injury. It must be repeated for an interval of six months to one year. These injuries may be potentially reversible during the first three postoperative months [8].

On the other hand, spinal cord and brain injuries are not described frequently in the literature. They may occur as a consequence of patient positioning during surgery or as a result of anesthetic techniques and drugs.

The beach chair position (BCP) is worldwide commonly used in shoulder surgery (open, percutaneous or arthroscopy) having more benefits than lateral decubitus position due to lower incidence of peripheral neurapraxia secondary to traction than in lateral decubitus position. Other described advantages are shorter operative time or easier, potential possibility to switch into open surgery an arthroscopic procedure if needed. Nevertheless, this position has been associated with brain and spinal cord stroke, midcervical quadriplegia, optic nerve lesions and higher risk of venous and arterial air embolism [9]. The etiology of these severe complications could be head and neck malpositioning accompanied by a cerebral hypoperfusion emphasized by hypotensive anesthesia used to reduce bleeding.

Interscalene block anesthesia is an alternative to general anesthesia in patients undergoing shoulder surgery. Their benefits are lower concentrations of anesthetics drugs, lower postoperative analgesic demand and lower incidence of postoperative nausea. However, it has been related to hypotensive and bradycardic heart events after its administration. Complications rates related to this anesthetic technique vary between 1.4 and $2.3 \%$, and they include brachial plexus neurapraxia, mis-induction of spinal or epidural anesthesia, seizures, cardiac arrest and potential block of phrenic nerve, laryngeal nerve and sympathetic chain $[9,10]$.

Back to our case, our patient suffered a spinal cord ischemia with-hemorrhage, without a clear explanation. According to the related published evidence, this severe and rare complication could be explained by the $\mathrm{BCP}$, the interscalene block anesthesia, outstanding in our patient an isolated episode of hypotension during induction of anesthesia.

\section{Conclusion}

Despite of the increasing number of RSA performed in elderly patients with three and four-part PHFs, there is no clear consensus about obtaining better functional results or a quicker recovery than with conservative management, moreover, considering a higher rate of complications.
Despite its low incidence, it is important when deciding PHF management to take into account potential severe complications related to RSA like neurological injuries, which are increasing in the last years, especially with RSA due to its nonanatomical design [6].

Therefore, it remains necessary to develop more prospective, control-trials comparative studies to determine which is the best option of treatment in this type of fractures in elderly patients so that we could improve our results minimizing complications.

\section{References}

1. Rangan A, Handoll $H$, Brealey $S$, Jefferson L, Keding A, et al. (2015) Surgical vs nonsurgical treatment of adults with displaced fractures of the proximal humerus: The PROFHER randomized clinical trial. JAMA 313: 1037-1047.

2. Zumstein MA, Pinedo M, Old J, Boileau P (2011) Problems, complications, reoperations, and revisions in reverse total shoulder arthroplasty: A systematic review. J Shoulder Elbow Surg 20: 146-157.

3. Barco R, Savvidou OD, Sperling JW, Sanchez-Sotelo J, Cofield RH (2016) Complications in reverse shoulder arthroplasty. EFORT Open Rev 1: 72-80.

4. Holton J, Yousri T, Arealis G, Levy O (2017) The role of reverse shoulder arthroplasty in management of proximal humerus fractures with fracture sequelae: A systematic review of the literature. Orthop Rev (Pavia) 9: 6977.

5. Leschinger T, Hackl M, Buess E, Lappen S, Scaal M, et al. (2017) The risk of suprascapular and axillary nerve injury in reverse total shoulder arthroplasty: An anatomic study. Injury 48: 2042-2049.

6. Ball CM (2017) Neurologic complications of shoulder joint replacement. J Shoulder Elbow Surg 26: 2125-2132.

7. Jauregui JJ, Nadarajah V, Shield WP 3rd, Henn RF 3rd, Gilotra M, et al. (2018) Reverse shoulder arthroplasty: Perioperative considerations and complications. JBJS Rev 6: e3.

8. Zhou HS, Chung JS, Yi PH, Li X, Price MD (2015) Management of complications after reverse shoulder arthroplasty. Curr Rev Musculoskelet Med 8: 92-97.

9. Rains DD, Rooke GA, Wahl CJ (2011) Pathomechanisms and complications related to patient positioning and anesthesia during shoulder arthroscopy. Arthroscopy 27: 532541.

10. Salazar DH, Davis WJ, Ziroğlu N, Garbis NG (2019) Cerebral desaturation events during shoulder arthroscopy in the beach chair position. J Am Acad Orthop Surg Glob Res Rev 3: e007. 